\title{
A STOCHASTIC FAILURE MODEL WITH DEPENDENT COMPETING RISKS AND ITS APPLICATIONS TO CONDITION-BASED MAINTENANCE
}

\author{
JI HWAN CHA, ${ }^{*}$ Ewha Womans University \\ INMA T. CASTRO, ${ }^{* *}$ University of Extremadura
}

\begin{abstract}
In this paper a stochastic failure model for a system with stochastically dependent competing failures is analyzed. The system is subject to two types of failure: degradation failure and catastrophic failure. Both types of failure share an initial common source: an external shock process. This implies that they are stochastically dependent. In our developments of the model, the type of dependency between the two kinds of failure will be characterized. Conditional properties of the two competing risks are also investigated. These properties are the fundamental basis for the development of the maintenance strategy studied in this paper. Considering this maintenance strategy, the long-run average cost rate is derived and the optimal maintenance policy is discussed.
\end{abstract}

Keywords: Dependent competing risks; degradation process; catastrophic failure; external shock process; baseline failure rate function; condition-based maintenance

2010 Mathematics Subject Classification: Primary 60K10

Secondary $62 \mathrm{P} 30$

\section{Introduction}

The mathematical sophistication of preventive maintenance models has increased in parallel to the growth in the complexity of the stochastic models under study. Surveys on various maintenance models from a practical point of view can be found in, e.g. Sherif and Smith (1981), Valdez-Flores and Feldman (1989), and Wang (2002). An overview on the maintenance theory from a theoretical point of view can be found in Nakagawa (2005). More theoretical and sophisticated models have also been developed (see Mi (1994), Aven (1996), Cha (2001), Ebrahimi (1997), and Aven and Jensen (1999)).

It is well known that most systems degrade physically over time. This degradation is due to the irreversible accumulation of damage through life and may involve corrosion, material fatigue, wearing out, and fracturing (Bogdanoff and Kozin (1985)). Such degradation process can be modelled by using a stochastic process and, frequently, the system is regarded as failed when its degradation first reaches a critical threshold level. In the literature on degradationbased stochastic failure models, frequently the degradation process is considered the only cause of failure (see, e.g. Castanier et al. (2003), Grall et al. (2002), Park and Padgett (2006), and Liao et al. (2006)). However, in many practical situations the systems can fail due to more

Received 14 January 2014; revision received 20 May 2014.

* Postal address: Department of Statistics, Ewha Womans University, Seoul, 120-750, Korea.

Email address: jhcha@ewha.ac.kr

** Postal address: Department of Mathematics, University of Extremadura, 10003 Cáceres, Spain.

Email address: inmatorres@unex.es 
than one cause. Furthermore, the types of failure can stochastically be dependent due to many different reasons.

In this paper we develop a stochastic failure model and perform subsequent analysis for the lifetime of a system working in a dynamic random environment with two 'stochastically dependent' competing risks: degradation and catastrophic failures. The analysis performed in this paper gives new insight into the structure of the stochastic dependency of the model and it provides a general theoretical basis for developing a maintenance policy under dependent competing risks. Then based on this insight, we develop a detailed preventive maintenance policy for the system. The topic will be discussed under a general setting, i.e. under a general stochastic process for the degradation process, not specifying it. Therefore, the results obtained in this paper are very general and this is one of the important contributions of this paper.

The structure of this paper is as follows. In Section 2 a stochastic failure model for a system under a dynamic random environment with two types of failure is developed. The joint survival function for the two competing risks will be derived and the type of dependency is characterized. In Section 3 the conditional properties of the two competing risks will be discussed, and the basis for the development of the preventive maintenance policy to be analyzed will be built. In Section 4, considering a maintenance policy for the system, we derive the long-run average cost rate function, and discuss the optimal maintenance policy based on it. In Section 5 we present a numerical example of this maintenance strategy. Finally, concluding remarks are given in Section 6.

\section{Stochastic model and dependency}

We start this section by suggesting a stochastic failure model for the system lifetime with two dependent competing risks. The system is operated under a random external shock process $\{N(t), t \geq 0\}$, where $N(t)$ denotes the number of shocks by the time $t$. The sequential arrival points of the shocks are denoted by $0 \leq T_{1} \leq T_{2} \leq \cdots$, where $T_{i}$ is the arrival time of the $i$ th shock, $i=1,2, \ldots$. Throughout this paper, we will assume that the external shock process $\{N(t), t \geq 0\}$ follows a nonhomogeneous Poisson process with intensity function $\lambda(t)$. As mentioned above, there are two types of failure. Let $Y$ be the time to a catastrophic failure (Type I) and let $S_{\mathrm{c}}$ be the time to a degradation failure (Type II).

Before describing the lifetime of the system under study, the introduction of the conditional failure rate 'under a general setting' is needed. We refer the reader to, e.g. Kalbfleisch and Prentice (1980), Aven and Jensen (1999), and Finkelstein and Cha (2013). Assume that a system, whose lifetime is denoted by $T$, is operating in a random environment described by a certain (covariate) stochastic process $\{Z(t), t \geq 0\}$. For example, the stochastic process $\{Z(t), t \geq 0\}$ can represent the randomly changing time-dependent external temperature, electric or mechanical load, or some other randomly changing external stress. Then the conditional failure rate can formally be defined as (see Kalbfleisch and Prentice (1980))

$$
r(t \mid z(u), 0 \leq u \leq t) \equiv \lim _{\Delta t \rightarrow 0} \frac{\mathbb{P}\{t<T \leq t+\Delta t \mid Z(u)=z(u), 0 \leq u \leq t, T>t\}}{\Delta t}
$$

where $\mathbb{P}$ is the probability measure. Note that this conditional failure rate can be specified for a realization of the covariate process. With the covariate process not fixed yet, it is also obviously a stochastic process, which is usually referred to as the 'failure (hazard) rate process' (or random failure rate). For details, see, e.g. Kebir (1991) and Aven and Jensen (1999). Then 
based on the failure (hazard) rate process the conditional survival function is given by

$$
\mathbb{P}\{T>t \mid Z(u)=z(u), 0 \leq u \leq t\}=\exp \left\{-\int_{0}^{t} r(s \mid z(u), 0 \leq u \leq s) \mathrm{d} s\right\} .
$$

We will now describe the lifetime distribution of our system. First, we will describe the conditional survival function of $Y$ given the shock process $\{N(t), t \geq 0\}$. We assume that an external shock at time $t$ provokes an immediate catastrophic failure with probability $p(t)$, or, with probability $q(t)=1-p(t)$, increases the failure rate function of $Y$ by $\eta$. Hence, given the shock process, the failure rate function of $Y$ is

$$
\begin{aligned}
r(t \mid n(u), 0 \leq u \leq t) & \equiv \lim _{\Delta t \rightarrow 0} \frac{\mathbb{P}\{t<Y \leq t+\Delta t \mid N(u)=n(u), 0 \leq u \leq t, Y>t\}}{\Delta t} \\
& =r_{0}(t)+\eta n(t),
\end{aligned}
$$

where $\eta>0$ and $r_{0}(t)$ is the 'baseline failure rate' which defines the lifetime distribution under a laboratory environment, i.e. in the absence of external shocks. We interpret (1) as follows: 'on each shock, the failure rate of $Y$ is increased by $\eta$ '. Hence, the external shocks increase the susceptibility to a catastrophic failure. By (1), the conditional survival function of $Y$ at time $t$, given the shock process and given no immediate catastrophic failure before time $t$, is given by

$\mathbb{P}\left\{Y>t \mid N(u), 0 \leq u \leq t, J_{i}=0, i=1, \ldots, N(t)\right\}=\exp \left\{-\int_{0}^{t} r_{0}(s) \mathrm{d} s-\eta \sum_{j=1}^{N(t)}\left(t-T_{j}\right)\right\}$,

where $J_{i}=1$ if the $i$ th shock provokes an immediate catastrophic failure and $J_{i}=0$ otherwise, $i=1,2, \ldots$.

We will now describe the conditional distribution of $S_{\mathrm{c}}$, given the shock process. We assume that at the arrival of each shock it instantly initiates an independent degradation process in the system. Therefore, at time $t, N(t)$ degradation processes are evolving in the system independently. We assume that the stochastic properties of the degradation process depend on its starting point $v$. We denote by $\left\{X_{v}(t), t \geq 0\right\}$ the nondecreasing degradation process initiated at time $v$, where $X_{v}(t)$ represents the deterioration level of the degradation process at $t$ time units after its initiation point $v$. Thus, degradation processes with different starting points do not necessarily follow the same type of process. For example, the degradation rate of the process can be increasing as the starting point increases, which can represent the effect of the system deterioration. For this kind of modelling specifically, the starting pointdependent nonhomogeneous gamma process could be employed. However, in the following discussions the results will be obtained without specifying the probability distribution of the degradation processes. Throughout this paper, we will only assume that $\left\{X_{v}(t), t \geq 0\right\}$ fulfills the independent increments property, but we will derive all the analytical results in this paper without specifying any other condition on the degradation process. We assume that the system fails when one of the degradation processes first reaches a critical threshold level $\xi_{\mathrm{c}}>0$. Let $f_{X_{v}(t)}(x)$ be the probability density function (PDF) of $X_{v}(t)$. Define $\sigma_{\mathrm{c}}(v)$ as the time from $v$ until the degradation, initiated at $v$, reaches the threshold level $\xi_{\mathrm{c}}$ and

$$
\bar{G}_{v}\left(t ; \xi_{\mathrm{c}}\right) \equiv \mathbb{P}\left\{\sigma_{\mathrm{c}}(v)>t\right\}=\mathbb{P}\left\{X_{v}(t) \leq \xi_{\mathrm{c}}\right\}=\int_{0}^{\xi_{\mathrm{c}}} f_{X_{v}(t)}(x) \mathrm{d} x
$$


Based on the above setting, the conditional survival function of $S_{\mathrm{c}}$ is obviously given by

$$
\mathbb{P}\left\{S_{\mathrm{c}}>t \mid N(u), 0 \leq u \leq t\right\}=\prod_{k=1}^{N(t)} \bar{G}_{T_{k}}\left(t-T_{k} ; \xi_{\mathrm{c}}\right),
$$

where, by convention, $\prod_{k=1}^{0}(\cdot) \equiv 1$. Finally, at this stage, we point out that, given the shock process, the two types of failure are 'conditionally independent'.

The above described model can be applied to complex systems composed of electrical and mechanical parts. In this case, external shocks can cause a catastrophic failure of the electrical part, whereas, at the same time, they can provoke the degradation processes of the mechanical part. As an example, aircraft jet engines are composed of electrical parts, and mechanical parts such as engine compressor blades. Furthermore, aircraft engines are subject to external shocks (vibrations) during take off, cruising, and landing.

We are now ready to derive the unconditional joint survival function of $Y$ and $S_{\mathrm{c}}$.

Theorem 1. The joint survival function of $Y$ and $S_{\mathrm{c}}$ is given by

$$
\begin{aligned}
\mathbb{P}\left\{Y>t, S_{\mathrm{c}}>u\right\}=\mathbf{1}_{\{t \geq u\}}[ & \exp \left\{-\int_{0}^{t} r_{0}(s) \mathrm{d} s\right\} \\
\times & \exp \left\{-\int_{0}^{u}\left(1-q(x) \exp \{-\eta(t-x)\} \bar{G}_{x}\left(u-x ; \xi_{\mathrm{c}}\right)\right) \lambda(x) \mathrm{d} x\right\} \\
\times & \left.\exp \left\{-\int_{u}^{t}(1-q(x) \exp \{-\eta(t-x)\}) \lambda(x) \mathrm{d} x\right\}\right] \\
+\mathbf{1}_{\{t<u\}}[ & \exp \left\{-\int_{0}^{t} r_{0}(s) \mathrm{d} s\right\} \\
& \times \exp \left\{-\int_{0}^{t}\left(1-q(x) \exp \{-\eta(t-x)\} \bar{G}_{x}\left(u-x ; \xi_{\mathrm{c}}\right)\right) \lambda(x) \mathrm{d} x\right\} \\
& \left.\times \exp \left\{-\int_{t}^{u}\left(1-\bar{G}_{x}\left(u-x ; \xi_{\mathrm{c}}\right)\right) \lambda(x) \mathrm{d} x\right\}\right],
\end{aligned}
$$

where $\mathbf{1}_{\{\cdot\}}$ denotes the indicator function.

Proof. (i) For the $t \geq u$ case. Note that, due to the conditional independence of $Y$ and $S_{\mathrm{c}}$,

$$
\begin{aligned}
\mathbb{P}\{Y> & \left.t, S_{\mathrm{c}}>u \mid N(s), 0 \leq s \leq t\right\} \\
= & \mathbb{P}\{Y>t \mid N(s), 0 \leq s \leq t\} \mathbb{P}\left\{S_{\mathrm{c}}>u \mid N(s), 0 \leq s \leq t\right\} \\
= & \mathbb{P}\left\{Y>t \mid N(s), 0 \leq s \leq t, J_{i}=0, i=1, \ldots, N(t)\right\} \\
& \times \mathbb{P}\left\{J_{i}=0, i=1, \ldots, N(t) \mid N(s), 0 \leq s \leq t\right\} \mathbb{P}\left\{S_{\mathrm{c}}>u \mid N(s), 0 \leq s \leq t\right\} \\
= & \prod_{i=1}^{N(t)} q\left(T_{i}\right) \exp \left\{-\int_{0}^{t} r_{0}(s) \mathrm{d} s-\eta \sum_{j=1}^{N(t)}\left(t-T_{j}\right)\right\} \prod_{k=1}^{N(u)} \bar{G}_{T_{k}}\left(u-T_{k} ; \xi_{\mathrm{c}}\right) \\
= & \exp \left\{-\int_{0}^{t} r_{0}(s) \mathrm{d} s\right\} \prod_{i=1}^{N(u)}\left(q\left(T_{i}\right) \exp \left\{-\eta\left(t-T_{i}\right)\right\} \bar{G}_{T_{i}}\left(u-T_{i} ; \xi_{\mathrm{c}}\right)\right) \\
& \quad \times \quad \prod_{k=1}^{N(t)-N(u)} q\left(V_{k}\right) \exp \left\{-\eta\left(t-V_{k}\right)\right\},
\end{aligned}
$$


where $V_{k}$ denotes the arrival time of the $k$ th shock in

$$
(u, t], u<V_{1} \leq V_{2} \leq \cdots \leq V_{N(t)-N(u)} \leq t .
$$

Then the joint survival function can be obtained by

$$
\begin{aligned}
& \mathbb{P}\left\{Y>t, S_{\mathrm{c}}>u\right\} \\
&=\exp \left\{-\int_{0}^{t} r_{0}(s) \mathrm{d} s\right\} \mathbb{E}\left\{\prod_{i=1}^{N(u)}\left(q\left(T_{i}\right) \exp \left\{-\eta\left(t-T_{i}\right)\right\} \bar{G}_{T_{i}}\left(u-T_{i} ; \xi_{\mathrm{c}}\right)\right)\right. \\
&\left.\times \prod_{k=1}^{N(t)-N(u)} q\left(V_{k}\right) \exp \left\{-\eta\left(t-V_{k}\right)\right\}\right\} .
\end{aligned}
$$

In (2), the expectation can be obtained by

$$
\begin{gathered}
\mathbb{E}\left\{\prod_{i=1}^{N(u)}\left(q\left(T_{i}\right) \exp \left\{-\eta\left(t-T_{i}\right)\right\} \bar{G}_{T_{i}}\left(u-T_{i} ; \xi_{\mathrm{c}}\right)\right) \prod_{k=1}^{N(t)-N(u)} q\left(V_{k}\right) \exp \left\{-\eta\left(t-V_{k}\right)\right\}\right\} \\
=\mathbb{E}\left\{\mathbb { E } \left\{\prod_{i=1}^{N(u)}\left(q\left(T_{i}\right) \exp \left\{-\eta\left(t-T_{i}\right)\right\} \bar{G}_{T_{i}}\left(u-T_{i} ; \xi_{\mathrm{c}}\right)\right)\right.\right. \\
\left.\left.\times \prod_{k=1}^{N(t)-N(u)} q\left(V_{k}\right) \exp \left\{-\eta\left(t-V_{k}\right)\right\} \mid N(u), N(t)-N(u)\right\}\right\} .
\end{gathered}
$$

In order to obtain the conditional expectation in (3), it is necessary to derive the conditional joint distribution of $\left(T_{1}, T_{2}, \cdots, T_{N(u)}, V_{1}, V_{2}, \cdots, V_{N(t)-N(u)} \mid N(u), N(t)-N(u)\right)$. Observe that the joint distribution of $\left(T_{1}, T_{2}, \cdots, T_{N(u)}, V_{1}, V_{2}, \cdots, V_{N(t)-N(u)}, N(u), N(t)-N(u)\right)$ is given by

$$
\begin{gathered}
f_{T_{1}, T_{2}, \cdots, T_{N(u)}, V_{1}, V_{2}, \cdots, V_{N(t)-N(u)}, N(u), N(t)-N(u)}\left(t_{1},, t_{2}, \cdots, t_{n}, v_{1}, v_{2}, \cdots, v_{m}, n, m\right) \\
=\left[\prod_{i=1}^{n} \lambda\left(t_{i}\right)\right] \exp \left\{-\int_{0}^{u} \lambda(w) \mathrm{d} w\right\}\left[\prod_{i=1}^{m} \lambda\left(v_{i}\right)\right] \exp \left\{-\int_{u}^{t} \lambda(w) \mathrm{d} w\right\}
\end{gathered}
$$

for $0 \leq t_{1} \leq t_{2} \leq \cdots \leq t_{n} \leq u \leq v_{1} \leq v_{2} \leq \cdots \leq t, n \geq 0, m \geq 0$. As $N(u)$ and $N(t)-N(u)$ follow independent Poisson distributions with means $\Lambda(u) \equiv \int_{0}^{u} \lambda(w) \mathrm{d} w$ and $\Lambda(t)-\Lambda(u)=\int_{u}^{t} \lambda(w) \mathrm{d} w$, respectively, the conditional distribution of

$$
\left(T_{1}, T_{2}, \cdots, T_{N(u)}, V_{1}, V_{2}, \cdots, V_{N(t)-N(u)} \mid N(u), N(t)-N(u)\right)
$$

is given by

$$
\begin{aligned}
& f_{T_{1}, T_{2}, \cdots, T_{N(u)}, V_{1}, V_{2}, \cdots, V_{N(t)-N(u)} \mid N(u), N(t)-N(u)}\left(t_{1}, t_{2}, \cdots, t_{n}, v_{1}, v_{2}, \cdots, v_{m} \mid n, m\right) \\
& \quad=n ! m !\left[\prod_{i=1}^{n} \frac{\lambda\left(t_{i}\right)}{\Lambda(u)}\right]\left[\prod_{i=1}^{m} \frac{\lambda\left(v_{i}\right)}{\Lambda(t)-\Lambda(u)}\right]
\end{aligned}
$$




$$
\begin{aligned}
& \text { for } 0 \leq t_{1} \leq t_{2} \leq \cdots \leq t_{n} \leq u \leq v_{1} \leq v_{2} \leq \cdots \leq t \text {. Then, we have } \\
& \qquad \begin{array}{l}
\mathbb{E}\left\{\prod_{i=1}^{N(u)}\left(q\left(T_{i}\right) \exp \left\{-\eta\left(t-T_{i}\right)\right\} \bar{G}_{T_{i}}\left(u-T_{i} ; \xi_{\mathrm{c}}\right)\right)\right. \\
\left.\quad \times \prod_{k=1}^{N(t)-N(u)} q\left(V_{k}\right) \exp \left\{-\eta\left(t-V_{k}\right)\right\} \mid N(u)=n, N(t)-N(u)=m\right\} \\
=\left(\int_{0}^{u} q(x) \exp \{-\eta(t-x)\} \bar{G}_{x}\left(u-x ; \xi_{\mathrm{c}}\right) \frac{\lambda(x)}{\Lambda(u)} \mathrm{d} x\right)^{n} \\
\quad \times\left(\int_{u}^{t} q(x) \exp \{-\eta(t-x)\} \frac{\lambda(x)}{(\Lambda(t)-\Lambda(u))} \mathrm{d} x\right)^{m} .
\end{array}
\end{aligned}
$$

Then, finally, the result can be obtained from (2) and (3).

(ii) For the $t<u$ case. In this case, by similar arguments to (i), we have the desired result.

From Theorem 1, the survival function of the system is given by

$$
\begin{aligned}
\mathbb{P}\left\{Y>t, S_{\mathrm{c}}>t\right\}= & \exp \left\{-\int_{0}^{t} r_{0}(s) \mathrm{d} s\right\} \\
& \times \exp \left\{-\int_{0}^{t}\left(1-q(x) \exp \{-\eta(t-x)\} \bar{G}_{x}\left(t-x ; \xi_{\mathrm{c}}\right)\right) \lambda(x) \mathrm{d} x\right\}
\end{aligned}
$$

for $t \geq 0$. Since the two competing risks share the common shock process, we will show that they are stochastically dependent. This dependency will properly constitute the fundamental basis for the preventive maintenance policy analyzed in the subsequent section. Thus, at this stage, it is important to characterize the type of stochastic dependency of the two competing risks. For our discussion, we need to define the concept of positive quadrant dependency.

Definition 1. Two random variables $T$ and $U$ are positively quadrant dependent (PQD) if the inequality

$$
\mathbb{P}\{T>t, U>u\} \geq \mathbb{P}\{T>t\} \mathbb{P}\{U>u\}
$$

holds for all $t$ and $u$.

It can be shown that if $T$ and $U$ are PQD then $\operatorname{cov}(T, U) \geq 0$ (Lehmann, 1966). Thus, the $\mathrm{PQD}$ is a stronger type of dependency than the positive covariance.

In Theorem 2, we show that the random variables $Y$ and $S_{\mathrm{c}}$ are PQD.

Theorem 2. The random variables $Y$ and $S_{\mathrm{c}}$ are $P Q D$, i.e.

$$
\mathbb{P}\left\{Y>t, S_{\mathrm{c}}>u\right\} \geq \mathbb{P}\{Y>t\} \mathbb{P}\left\{S_{\mathrm{c}}>u\right\} \text { for all } t \text { and } u
$$


Proof. (i) For the $t \geq u$ case. From Theorem 1, we have

$$
\begin{aligned}
& \mathbb{P}\left\{Y>t, S_{\mathrm{c}}>u\right\}-\mathbb{P}\{Y>t\} \mathbb{P}\left\{S_{\mathrm{c}}>u\right\} \\
&=\exp \left\{-\int_{0}^{t} r_{0}(s) \mathrm{d} s\right\} \exp \left\{-\int_{u}^{t}(1-q(x) \exp \{-\eta(t-x)\}) \lambda(x) \mathrm{d} x\right\} \\
& \times {\left[\exp \left\{-\int_{0}^{u}\left(1-q(x) \exp \{-\eta(t-x)\} \bar{G}_{x}\left(u-x ; \xi_{\mathrm{c}}\right)\right) \lambda(x) \mathrm{d} x\right\}\right.} \\
&\left.\quad-\exp \left\{-\int_{0}^{u}\left[(1-q(x) \exp \{-\eta(t-x)\})+\left(1-\bar{G}_{x}\left(u-x ; \xi_{\mathrm{c}}\right)\right)\right] \lambda(x) \mathrm{d} x\right\}\right] .
\end{aligned}
$$

In the above equation,

$$
\begin{aligned}
& \left(1-q(x) \exp \{-\eta(t-x)\} \bar{G}_{x}\left(u-x ; \xi_{\mathrm{c}}\right)\right) \\
& -\left[(1-q(x) \exp \{-\eta(t-x)\})+\left(1-\bar{G}_{x}\left(u-x ; \xi_{\mathrm{c}}\right)\right)\right] \\
& \quad=-G_{x}\left(u-x ; \xi_{\mathrm{c}}\right)(1-q(x) \exp \{-\eta(t-x)\}) \\
& \quad \leq 0 \quad \text { for all } 0 \leq x \leq u,
\end{aligned}
$$

where $G_{x}\left(u-x ; \xi_{\mathrm{c}}\right) \equiv 1-\bar{G}_{x}\left(u-x ; \xi_{\mathrm{c}}\right)$. Therefore, we have

$$
\mathbb{P}\left\{Y>t, S_{\mathrm{c}}>u\right\} \geq \mathbb{P}\{Y>t\} \mathbb{P}\left\{S_{\mathrm{c}}>u\right\} \quad \text { for all } t \geq u .
$$

(ii) For the $t<u$ case. In this case, similar to (i), it can be shown without difficulty that $\mathbb{P}\left\{Y>t, S_{\mathrm{c}}>u\right\} \geq \mathbb{P}\{Y>t\} \mathbb{P}\left\{S_{\mathrm{c}}>u\right\}$ for all $t<u$.

\section{Conditional properties of the competing risks}

In this section we will analyze some conditional properties of the two competing risks. These properties constitute the fundamental basis for the preventive maintenance policy applied to this system. It is clear that the susceptibility to failure of our system increases as the system suffers more and more external shocks. Therefore, if the shock process can be observed then this information could be used for determining the preventive maintenance policy. For example, suppose that the system is currently working at time $t$, but it is known that it has experienced a large number of shocks in $(0, t]$. Then in order to prevent a system failure, it can be replaced by a new one. However, in most practical cases, the shock process cannot be observed. Then in order to obtain information on the system status, an inspection can be performed at time $t$. In these inspection times, the degradation levels of the degradation processes can be measured. If the degradation level of a process exceeds a preventive threshold $\xi_{\mathrm{p}}$, the system is replaced by a new one. Otherwise, the system continues working without being replaced.

Note that the above procedure utilizes the information directly related with Type II (degradation failure) and, accordingly, it would contribute to the prevention of a failure due to Type II. However, as the two types are stochastically dependent (positively dependent), this procedure would also contribute to the prevention of an unexpected failure due to Type I (catastrophic failure), and eventually it would improve the (residual) survival probability of the system. This reasoning is verified in Theorem 3. The first two results of Theorem 3 show the contribution of the preventive maintenance strategy described above to marginal types of failure, and the last result shows its contribution to the survival probability of the system. A more detailed interpretation will be given just after the theorem. 
For our discussions, we need to introduce some notation. We define $S_{\mathrm{p}}$ as the time instant at which any one of the degradation processes exceed the preventive threshold level $\xi_{\mathrm{p}}$ for the first time. Furthermore, let us define

$$
\bar{G}_{v}\left(t ; \xi_{\mathrm{p}}\right) \equiv \mathbb{P}\left\{X_{v}(t) \leq \xi_{\mathrm{p}}\right\}, \quad \bar{G}_{v}\left(s, t ; \xi_{\mathrm{p}}, \xi_{\mathrm{c}}\right) \equiv \mathbb{P}\left\{X_{v}(s) \leq \xi_{\mathrm{p}}, X_{v}(t) \leq \xi_{\mathrm{c}}\right\}
$$

Then as the degradation process $\left\{X_{v}(t), t \geq 0\right\}$ possesses the independent increments property, we have

$$
\begin{aligned}
\bar{G}_{v}\left(s, t ; \xi_{\mathrm{p}}, \xi_{\mathrm{c}}\right) & =\int_{0}^{\xi_{\mathrm{p}}} \mathbb{P}\left\{X_{v}(t)-X_{v}(s) \leq \xi_{\mathrm{c}}-x\right\} f_{X_{v}(s)}(x) \mathrm{d} x \\
& =\int_{0}^{\xi_{\mathrm{p}}} F_{X_{v}(t)-X_{v}(s)}\left(\xi_{c}-x\right) f_{X_{v}(s)}(x) \mathrm{d} x
\end{aligned}
$$

where $F_{X_{v}(t)-X_{v}(s)}(x)$ denotes the cumulative distribution function of $X_{v}(t)-X_{v}(s)$.

Theorem 3. Suppose that the degradation process $\left\{X_{v}(t), t \geq 0\right\}$ fulfills the independent increments property. Then the following properties hold:

(i) $\mathbb{P}\left\{Y>t+s \mid Y>t, S_{\mathrm{p}}>t\right\} \geq \mathbb{P}\{Y>t+s \mid Y>t\}$ for all $t \geq 0, s \geq 0$.

(ii) $\mathbb{P}\left\{S_{\mathrm{c}}>t+s \mid S_{\mathrm{c}}>t, S_{\mathrm{p}}>t\right\} \geq \mathbb{P}\left\{S_{\mathrm{c}}>t+s \mid S_{\mathrm{c}}>t\right\}$ for all $t \geq 0, s \geq 0$.,

(iii) $\mathbb{P}\left\{Y>t+s, S_{\mathrm{c}}>t+s \mid Y>t, S_{\mathrm{c}}>t, S_{\mathrm{p}}>t\right\} \geq \mathbb{P}\left\{Y>t+s, S_{\mathrm{c}}>t+s \mid Y>t\right.$, $\left.S_{\mathrm{c}}>t\right\}$ for all $t \geq 0, s \geq 0$.

Proof. Observe that in property (iii) if we let $\xi_{\mathrm{c}} \equiv \infty$, we can obtain property (i); and if we let $p(t) \equiv 0$ for all $t \geq 0$ and $\eta \equiv 0$, then we can obtain property (ii). Therefore, it is sufficient to show property (iii). From (4),

$$
\begin{aligned}
\mathbb{P}\{Y> & \left.t+s, S_{\mathrm{c}}>t+s \mid Y>t, S_{\mathrm{c}}>t\right\} \\
= & \exp \left\{-\int_{t}^{t+s} r_{0}(s) \mathrm{d} s\right\} \exp \left\{\int _ { 0 } ^ { t } \left(q(x) \exp \{-\eta(t+s-x)\} \bar{G}_{x}\left(t+s-x ; \xi_{\mathrm{c}}\right)\right.\right. \\
& \left.\left.-q(x) \exp \{-\eta(t-x)\} \bar{G}_{x}\left(t-x ; \xi_{\mathrm{c}}\right)\right) \lambda(x) \mathrm{d} x\right\} \\
& \times \exp \left\{-\int_{t}^{t+s}\left(1-q(x) \exp \{-\eta(t+s-x)\} \bar{G}_{x}\left(t+s-x ; \xi_{\mathrm{c}}\right)\right) \lambda(x) \mathrm{d} x\right\} .
\end{aligned}
$$

We will now derive $\mathbb{P}\left\{Y>t+s, S_{\mathrm{c}}>t+s \mid Y>t, S_{\mathrm{c}}>t, S_{\mathrm{p}}>t\right\}$. Thus,

$$
\begin{aligned}
\mathbb{P}\{Y> & \left.t+s, S_{\mathrm{c}}>t+s \mid Y>t, S_{\mathrm{c}}>t, S_{\mathrm{p}}>t\right\} \\
= & \mathbb{P}\left\{Y>t+s, S_{\mathrm{c}}>t+s \mid Y>t, S_{\mathrm{p}}>t\right\} \\
= & \mathbb{E}_{\left\{N(t), N(t+s)-N(t) \mid Y>t, S_{\mathrm{p}}>t\right\}} \\
& \left\{\mathbb{P}\left\{Y>t+s, S_{\mathrm{c}}>t+s \mid Y>t, S_{\mathrm{p}}>t, N(t), N(t+s)-N(t)\right\}\right\} .
\end{aligned}
$$

Here,

$$
\begin{aligned}
\mathbb{P}\left\{Y>t+s, S_{\mathrm{c}}>t+s \mid Y>t, S_{\mathrm{p}}>t, N(t), N(t+s)-N(t)\right\} \\
=\mathbb{E}_{\left\{T_{i}^{\prime} s, V_{i}^{\prime} s \mid Y>t, S_{\mathrm{p}}>t, N(t), N(t+s)-N(t)\right\}} \\
\left\{\mathbb { P } \left\{Y>t+s, S_{\mathrm{c}}>t+s \mid Y>t, S_{\mathrm{p}}>t, N(t), T_{1}, T_{2}, \cdots, T_{N(t)},\right.\right. \\
\left.\left.\quad N(t+s)-N(t), V_{1}, V_{2}, \cdots, V_{N(t+s)-N(t)}\right\}\right\},
\end{aligned}
$$


where $V_{i}$ is the arrival time of the $i$ th shock in $(t, t+s]$. Observe that

$$
\begin{aligned}
& \mathbb{P}\left\{Y>t+s, S_{\mathrm{c}}>t+s \mid Y>t, S_{\mathrm{p}}>t\right. \\
& \left.N(t), T_{1}, T_{2}, \cdots, T_{N(t)}, N(t+s)-N(t), V_{1}, V_{2}, \cdots, V_{N(t+s)-N(t)}\right\} \\
& =\exp \left\{-\int_{t}^{t+s} r_{0}(u)+N(t) \eta \mathrm{d} u\right\} \prod_{i=1}^{N(t)} \mathbb{P}\left\{X_{T_{i}}\left(t+s-T_{i}\right) \leq \xi_{\mathrm{c}} \mid X_{T_{i}}\left(t-T_{i}\right) \leq \xi_{\mathrm{p}}\right\} \\
& \quad \times \prod_{k=1}^{N(t+s)-N(t)} q\left(V_{i}\right) \exp \left\{-\eta\left(t+s-V_{i}\right)\right\} \bar{G}_{V_{i}}\left(t+s-V_{i} ; \xi_{\mathrm{c}}\right) .
\end{aligned}
$$

In order to take the conditional expectation in (6), we will now derive the conditional joint distribution of

$$
\left(T_{1}, T_{2}, \cdots, T_{N(t)}, V_{1}, V_{2}, \cdots, V_{N(t+s)-N(t)} \mid Y>t, S_{\mathrm{p}}>t, N(t), N(t+s)-N(t)\right) .
$$

Observe that the joint distribution of

$$
\left(Y>t, S_{\mathrm{p}}>t, N(t), N(t+s)-N(t), T_{1}, T_{2}, \cdots, T_{N(t)}, V_{1}, V_{2}, \cdots, V_{N(t+s)-N(t)}\right)
$$
is

$$
\begin{aligned}
& \exp \left\{-\int_{0}^{t} r_{0}(u) \mathrm{d} u\right\}\left(\prod_{i=1}^{n} q\left(t_{i}\right) \exp \left\{-\eta\left(t-t_{i}\right)\right\} \bar{G}_{t_{i}}\left(t-t_{i} ; \xi_{\mathrm{p}}\right)\right) \\
& \times \prod_{i=1}^{n} \lambda\left(t_{i}\right) \exp \left\{-\int_{0}^{t} \lambda(u) \mathrm{d} u\right\} \prod_{j=1}^{m} \lambda\left(v_{j}\right) \exp \left\{-\int_{t}^{t+s} \lambda(u) \mathrm{d} u\right\}
\end{aligned}
$$

for $0<t_{1}<t_{2}<\ldots<t_{n}<t$ and $t<v_{1}<v_{2}<\ldots<v_{m}<t+s$. Thus, the joint distribution of $\left(Y>t, S_{\mathrm{p}}>t, N(t), N(t+s)-N(t)\right)$ is

$$
\begin{aligned}
& \exp \left\{-\int_{0}^{t} r_{0}(u) \mathrm{d} u\right\} \frac{1}{n !}\left(\int_{0}^{t} q(x) \exp \{-\eta(t-x)\} \bar{G}_{x}\left(t-x ; \xi_{\mathrm{p}}\right) \lambda(x) \mathrm{d} x\right)^{n} \\
& \times \exp \{-\Lambda(t)\} \frac{1}{m !}\left(\int_{t}^{t+s} \lambda(u) \mathrm{d} u\right)^{m} \exp \{-(\Lambda(t+s)-\Lambda(t))\}
\end{aligned}
$$

Then the conditional joint distribution of

$$
\left(T_{1}, T_{2}, \cdots, T_{N(t)}, V_{1}, V_{2}, \cdots, V_{N(t+s)-N(t)} \mid Y>t, S_{\mathrm{p}}>t, N(t), N(t+s)-N(t)\right)
$$
is

$$
n !\left[\prod_{i=1}^{n} \frac{q\left(t_{i}\right) \exp \left\{-\eta\left(t-t_{i}\right)\right\} \bar{G}_{t_{i}}\left(t-t_{i} ; \xi_{\mathrm{p}}\right) \lambda\left(t_{i}\right)}{\int_{0}^{t} q(x) \exp \{-\eta(t-x)\} \bar{G}_{x}\left(t-x ; \xi_{\mathrm{p}}\right) \lambda(x) \mathrm{d} x}\right] m !\left[\prod_{k=1}^{m} \frac{\lambda\left(v_{k}\right)}{\Lambda(t+s)-\Lambda(t)}\right] .
$$

Thus,

$$
\begin{aligned}
\mathbb{P}\{Y> & \left.t+s, S_{\mathrm{c}}>t+s \mid Y>t, S_{\mathrm{p}}>t, N(t)=n, N(t+s)-N(t)=m\right\} \\
= & \exp \left\{-\int_{t}^{t+s} r_{0}(u) \mathrm{d} u\right\}(\exp \{-\eta s\})^{n} \\
& \times\left(\frac{\int_{0}^{t} q(x) \exp \{-\eta(t-x)\} \bar{G}_{x}\left(t-x, t+s-x ; \xi_{\mathrm{p}}, \xi_{\mathrm{c}}\right) \lambda(x) \mathrm{d} x}{\int_{0}^{t} q(x) \exp \{-\eta(t-x)\} \bar{G}_{x}\left(t-x ; \xi_{\mathrm{p}}\right) \lambda(x) \mathrm{d} x}\right)^{n} \\
& \times\left(\frac{\int_{t}^{t+s} q(x) \exp \{-\eta(t+s-x)\} \bar{G}_{x}\left(t+s-x ; \xi_{\mathrm{c}}\right) \lambda(x) \mathrm{d} x}{\Lambda(t+s)-\Lambda(t)}\right)^{m} .
\end{aligned}
$$


Now, in order to take the conditional expectation in (5), the conditional distribution of $\left(N(t), N(t+s)-N(t) \mid Y>t, S_{\mathrm{p}}>t\right)$ can be obtained from (7), which is given by

$$
\begin{aligned}
& \frac{1}{n !}\left(\int_{0}^{t} q(x) \exp \{-\eta(t-x)\} \bar{G}_{x}\left(t-x ; \xi_{\mathrm{p}}\right) \lambda(x) \mathrm{d} x\right)^{n} \\
& \quad \times \exp \left\{-\int_{0}^{t} q(x) \exp \{-\eta(t-x)\} \bar{G}_{x}\left(t-x ; \xi_{\mathrm{p}}\right) \lambda(x) \mathrm{d} x\right\} \\
& \quad \times \frac{1}{m !}\left(\int_{t}^{t+s} \lambda(u) \mathrm{d} u\right)^{m} \exp \{-(\Lambda(t+s)-\Lambda(t))\} .
\end{aligned}
$$

Therefore,

$$
\begin{aligned}
\mathbb{P}\{Y> & \left.t s, S_{\mathrm{c}}>t+s \mid Y>t, S_{\mathrm{p}}>t\right\} \\
= & \exp \left\{-\int_{t}^{t+s} r_{0}(u) \mathrm{d} u\right\} \\
\times & \exp \left\{\int _ { 0 } ^ { t } \left(q(x) \exp \{-\eta(t+s-x)\} \bar{G}_{x}\left(t-x, t+s-x ; \xi_{\mathrm{p}}, \xi_{\mathrm{c}}\right)-q(x)\right.\right. \\
& \left.\left.\times \exp \{-\eta(t-x)\} \bar{G}_{x}\left(t-x ; \xi_{\mathrm{p}}\right)\right) \lambda(x) \mathrm{d} x\right\} \\
& \times \exp \left\{-\int_{t}^{t+s}\left(1-q(x) \exp \{-\eta(t+s-x)\} \bar{G}_{x}\left(t+s-x ; \xi_{\mathrm{c}}\right)\right) \lambda(x) \mathrm{d} x\right\} .
\end{aligned}
$$

We will now compare $\mathbb{P}\left\{Y>t+s, S_{\mathrm{c}}>t+s \mid Y>t, S_{\mathrm{p}}>t\right\}$ with $\mathbb{P}\left\{Y>t+s, S_{\mathrm{c}}>\right.$ $\left.t+s \mid Y>t, S_{\mathrm{c}}>t\right\}$. For this, it is now sufficient to compare

$$
L_{1}(x) \equiv q(x) \exp \{-\eta(t+s-x)\} \bar{G}_{x}\left(t+s-x ; \xi_{\mathrm{c}}\right)-q(x) \exp \{-\eta(t-x)\} \bar{G}_{x}\left(t-x ; \xi_{\mathrm{c}}\right)
$$

and

$$
\begin{aligned}
L_{2}(x) \equiv & q(x) \exp \{-\eta(t+s-x)\} \bar{G}_{x}\left(t-x, t+s-x ; \xi_{\mathrm{p}}, \xi_{\mathrm{c}}\right)-q(x) \\
& \times \exp \{-\eta(t-x)\} \bar{G}_{x}\left(t-x ; \xi_{\mathrm{p}}\right) .
\end{aligned}
$$

Observe that

$$
\begin{aligned}
L_{1}(x)-L_{2}(x)= & q(x) \exp \{-\eta(t-x)\} \\
& \times\left(\exp \{-\eta s\}\left[\bar{G}_{x}\left(t+s-x ; \xi_{\mathrm{c}}\right)-\bar{G}_{x}\left(t-x, t+s-x ; \xi_{\mathrm{p}}, \xi_{\mathrm{c}}\right)\right]\right. \\
& \left.\quad-\left[\bar{G}_{x}\left(t-x ; \xi_{\mathrm{c}}\right)-\bar{G}_{x}\left(t-x ; \xi_{\mathrm{p}}\right)\right]\right) .
\end{aligned}
$$

Note here that

$$
\begin{aligned}
& {\left[\bar{G}_{x}\left(t+s-x ; \xi_{\mathrm{c}}\right)-\bar{G}_{x}\left(t-x, t+s-x ; \xi_{\mathrm{p}}, \xi_{\mathrm{c}}\right)\right]\left[\bar{G}_{x}\left(t-x, \xi_{\mathrm{c}}\right)-\bar{G}_{x}\left(t-x ; \xi_{\mathrm{p}}\right)\right]^{-1}} \\
& \quad=\frac{\mathbb{P}\left\{X_{x}(t+s-x) \leq \xi_{\mathrm{c}}\right\}-\mathbb{P}\left\{X_{x}(t-x) \leq \xi_{\mathrm{p}}, X_{x}(t+s-x) \leq \xi_{\mathrm{c}}\right\}}{\mathbb{P}\left\{X_{x}(t-x) \leq \xi_{\mathrm{c}}\right\}-\mathbb{P}\left\{X_{x}(t-x) \leq \xi_{\mathrm{p}}\right\}} \\
& \quad=\frac{\mathbb{P}\left\{X_{x}(t-x) \leq \xi_{\mathrm{c}}, X_{x}(t+s-x) \leq \xi_{\mathrm{c}}\right\}-\mathbb{P}\left\{X_{x}(t-x) \leq \xi_{\mathrm{p}}, X_{x}(t+s-x) \leq \xi_{\mathrm{c}}\right\}}{\mathbb{P}\left\{X_{x}(t-x) \leq \xi_{\mathrm{c}}\right\}-\mathbb{P}\left\{X_{x}(t-x) \leq \xi_{\mathrm{p}}\right\}} \\
& \quad=\frac{\mathbb{P}\left\{\xi_{\mathrm{p}}<X_{x}(t-x) \leq \xi_{\mathrm{c}}, X_{x}(t+s-x) \leq \xi_{\mathrm{c}}\right\}}{\mathbb{P}\left\{\xi_{\mathrm{p}}<X_{x}(t-x) \leq \xi_{\mathrm{c}}\right\}} \\
& \quad \leq 1 .
\end{aligned}
$$


The above inequality implies that $L_{1}(x)-L_{2}(x) \leq 0$ for $x \in[0, t]$ and, finally, we can now conclude that property (iii) holds.

As mentioned above, the first two results show the contribution of the preventive maintenance strategy to the marginal types of failure. For example, suppose that the system has survived to a catastrophic failure at time $t$. In this case, the residual lifetime until failure due to Type I is given by $\mathbb{P}\{Y>t+s \mid Y>t\}$. Furthermore, suppose that the replacement strategy described in the first part of this section is applied. Then, by this maintenance strategy, the systems with degradation processes exceeding $\xi_{\mathrm{p}}$ at time $t$ (i.e. the systems with $S_{\mathrm{p}} \leq t$ ) are eliminated and only the systems that fulfill $S_{\mathrm{p}}>t$ continue working. Thus, in this case, the residual lifetime until failure due to Type $\mathrm{I}$ is described by $\mathbb{P}\left\{Y>t+s \mid Y>t, S_{\mathrm{p}}>t\right\}$. In the first result of Theorem 3, it was shown that $\mathbb{P}\left\{Y>t+s \mid Y>t, S_{\mathrm{p}}>t\right\} \geq \mathbb{P}\{Y>t+s \mid Y>t\}$. As mentioned above, the preventive maintenance strategy utilizes information which is directly related to the Type II failure, but it also contributes to the prevention of an unexpected failure by Type I due to the stochastic dependence between them. The second result of Theorem 3 can also be interpreted in a similar way. For the interpretation of the last result of Theorem 3, we need to consider the two types at the same time. Suppose that the system survives at time $t$. In this case, the residual lifetime of the system is described by $\mathbb{P}\left\{Y>t+s, S_{\mathrm{c}}>t+s \mid Y>t, S_{\mathrm{c}}>t\right\}$. However, if the preventive maintenance strategy is applied, the residual lifetime is given by $\mathbb{P}\left\{Y>t+s, S_{\mathrm{c}}>t+s \mid Y>t, S_{\mathrm{c}}>t, S_{\mathrm{p}}>t\right\}$ and, by Theorem 3, the preventive maintenance strategy improves the residual survival probability of the system.

Therefore, we are now ready to discuss the preventive maintenance strategy suggested in this section more formally and systematically in the next section.

\section{Maintenance strategy}

In Section 3 the preventive maintenance policy based on the degradation level was fully justified. In this section the maintenance strategy is discussed in a more formal way.

We assume that the failure of the system can be detected only by an inspection, and the system is inspected each $T$ units of time to check its status. On each inspection, if the system is failed then the system is replaced by a new one (a corrective maintenance). If it is not, in these inspection times, the deterioration level of the degradation processes is measured. If the deterioration level of any degradation process exceeds a threshold $\xi_{\mathrm{p}}\left(0<\xi_{\mathrm{p}} \leq \xi_{\mathrm{c}}\right)$ then the system is replaced by a new one (a preventive maintenance); otherwise no maintenance task is performed. The time to perform a maintenance action is negligible.

We assume that the cost of a corrective replacement is $C_{\mathrm{c}}$ monetary units (MUs), the cost of a preventive replacement is $C_{\mathrm{p}}(\mathrm{MU})$, the corresponding cost for each inspection is $C_{\mathrm{I}}$ (MU) and the cost incurred by the downtime of the system is $C_{\mathrm{d}}(\mathrm{MU})$ per unit time. Now we will calculate some measures of interest related to this maintenance policy. Before doing so, we will calculate some survival functions used to obtain these measures.

We denote by $\bar{F}_{\min \left(Y, S_{\mathrm{c}}\right)}$ the survival function of $\min \left(Y, S_{\mathrm{c}}\right)$. By Theorem 1 ,

$$
\begin{aligned}
\bar{F}_{\min \left(Y, S_{\mathrm{c}}\right)}(t)= & \mathbb{P}\left\{Y>t, S_{\mathrm{c}}>t\right\} \\
= & \exp \left\{-\int_{0}^{t} r_{0}(s) \mathrm{d} s\right\} \\
& \times \exp \left\{-\int_{0}^{t}\left(1-q(x) \exp \{-\eta(t-x)\} \bar{G}_{x}\left(t-x ; \xi_{\mathrm{c}}\right)\right) \lambda(x) \mathrm{d} x\right\} .
\end{aligned}
$$


Analogously, we obtain the joint distribution of $\left(\min \left(Y, S_{\mathrm{c}}\right), S_{\mathrm{p}}\right)$. For $0 \leq u \leq t$,

$$
\begin{aligned}
\bar{F}_{1}(t, u)= & \mathbb{P}\left\{\min \left(Y, S_{\mathrm{c}}\right)>t, S_{\mathrm{p}}>u\right\} \\
= & \mathbb{P}\left\{Y>t, S_{\mathrm{c}}>t, S_{\mathrm{p}}>u\right\} \\
= & \exp \left\{-\int_{0}^{t} r_{0}(u) \mathrm{d} u\right\} \\
& \times \exp \left\{-\int_{0}^{u}\left(1-q(x) \exp \{-\eta(t-x)\} \bar{G}_{x}\left(u-x, t-x ; \xi_{\mathrm{p}}, \xi_{\mathrm{c}}\right)\right) \lambda(x) \mathrm{d} x\right\} \\
& \times \exp \left\{-\int_{u}^{t}\left(1-q(x) \exp \{-\eta(t-x)\} \bar{G}_{x}\left(t-x ; \xi_{\mathrm{c}}\right)\right) \lambda(x) \mathrm{d} x\right\},
\end{aligned}
$$

and for $0 \leq t \leq u$, we have

$$
\begin{aligned}
\bar{F}_{2}(t, u)= & \mathbb{P}\left\{\min \left(Y, S_{\mathrm{c}}\right)>t, S_{\mathrm{p}}>u\right\} \\
= & \mathbb{P}\left\{Y>t, S_{\mathrm{p}}>u\right\} \\
= & \exp \left\{-\int_{0}^{t} r_{0}(u) \mathrm{d} u\right\} \\
& \times \exp \left\{-\int_{0}^{t}\left(1-q(x) \exp \{-\eta(t-x)\} \bar{G}_{x}\left(u-x ; \xi_{\mathrm{p}}\right)\right) \lambda(x) \mathrm{d} x\right\} \\
& \left.\times \exp \left\{-\int_{t}^{u}\left(1-\bar{G}_{x}\left(u-x ; \xi_{\mathrm{p}}\right)\right)\right) \lambda(x) \mathrm{d} x\right\} .
\end{aligned}
$$

We denote by $f_{1}(t, u)$ and $f_{2}(t, u)$ the bidimensional density functions associated to $\bar{F}_{1}$ and $\bar{F}_{2}$, respectively, i.e. the functions that fulfill

$$
\begin{array}{ll}
\bar{F}_{1}(t, u)=\int_{t}^{\infty} \int_{u}^{\infty} f_{1}(w, s) \mathrm{d} s \mathrm{~d} w, & t \geq u, \\
\bar{F}_{2}(t, u)=\int_{t}^{\infty} \int_{u}^{\infty} f_{2}(w, s) \mathrm{d} s \mathrm{~d} w, & u \geq t .
\end{array}
$$

Probability of the maintenance actions. Let $\mathbb{P}_{\mathrm{p}}\{k T\}$ be the probability of a preventive maintenance action at time $k T$. A preventive maintenance action is performed at time $k T$, $k=1,2, \cdots$, if

$$
\left\{(k-1) T \leq S_{\mathrm{p}} \leq k T, k T \leq \min \left(S_{\mathrm{c}}, Y\right)\right\}
$$

occurs. Hence,

$$
\begin{aligned}
\mathbb{P}_{\mathrm{p}}\{k T\} & =\mathbb{P}\left\{(k-1) T \leq S_{\mathrm{p}} \leq k T, k T \leq \min \left(S_{\mathrm{c}}, Y\right)\right\} \\
& =\int_{(k-1) T}^{k T} \mathrm{~d} u \int_{k T}^{\infty} f_{1}(t, u) \mathrm{d} t, \quad k=1,2, \ldots, T>0,
\end{aligned}
$$

where $f_{1}$ is given by (8).

Let $\mathbb{P}_{c}\{k T\}$ be the probability of a corrective maintenance at $k T$ for $k=1,2, \ldots$. For this maintenance strategy, the failure is detected only by inspection, and the corrective maintenance is performed only at these points. Hence, a corrective replacement is performed at $T$ if $\left\{\min \left(Y, S_{\mathrm{c}}\right) \leq T\right\}$ occurs and, at $k T, k=2,3 \ldots$, if the following occurs:

$$
\left\{(k-1) T<S_{\mathrm{p}},(k-1) T<\min \left(Y, S_{\mathrm{c}}\right) \leq k T\right\} .
$$


Hence,

$$
\begin{aligned}
& \mathbb{P}_{\mathrm{c}}\{k T\}=\mathbb{P}\left\{\min \left(Y, S_{\mathrm{c}}\right) \leq T\right\} \mathbf{1}_{\{k=1\}} \\
& +\mathbb{P}\left\{(k-1) T<S_{\mathrm{p}},(k-1) T<\min \left(Y, S_{\mathrm{c}}\right) \leq k T\right\} \mathbf{1}_{\{k>1\}} \\
& =\mathbf{1}_{\{k=1\}} F_{\min \left(Y, S_{\mathrm{c}}\right)}(T)+\left(\int_{(k-1) T}^{k T} \mathrm{~d} u \int_{(k-1) T}^{u} f_{2}(t, u) \mathrm{d} t\right. \\
& +\int_{(k-1) T}^{k T} \mathrm{~d} u \int_{u}^{k T} f_{1}(t, u) \mathrm{d} t \\
& \left.+\int_{k T}^{\infty} \mathrm{d} u \int_{(k-1) T}^{k T} f_{2}(t, u) \mathrm{d} t\right) \mathbf{1}_{\{k>1\}} .
\end{aligned}
$$

Expected time to a replacement cycle. Let $R$ be the time to a replacement cycle under this maintenance strategy. Then $R=k T$ if

$$
\left\{(k-1) T<S_{\mathrm{p}} \leq k T, k T \leq \min \left(S_{\mathrm{c}}, Y\right)\right\},
$$

or

$$
\left\{(k-1) T<S_{\mathrm{p}},(k-1) T<\min \left(Y, S_{\mathrm{c}}\right)<k T\right\}
$$

for $k=1,2, \ldots$ Then

$$
\mathbb{E}\{R\}=\sum_{k=1}^{\infty} k T\left(\mathbb{P}_{\mathrm{p}}\{k T\}+\mathbb{P}_{\mathrm{c}}\{k T\}\right),
$$

where $\mathbb{P}_{\mathrm{p}}\{k T\}$ and $\mathbb{P}_{\mathrm{c}}\{k T\}$ are given by (9) and (10), respectively.

Expected number of inspections. We assume that the cost of inspection is mainly due to the measurement of the degradation levels, and, thus, if the system is failed then there is no incurred inspection cost on the corresponding inspection. Then the expected number of inspections involving inspection cost in a replacement cycle is given by

$$
\mathbb{E}\{N\}=\sum_{k=1}^{\infty} k\left(\mathbb{P}_{\mathrm{c}}\{(k+1) T\}+\mathbb{P}_{\mathrm{p}}\{k T\}\right),
$$

where $\mathbb{P}_{\mathrm{p}}\{k T\}$ and $\mathbb{P}_{\mathrm{c}}\{k T\}$ are given by (9) and (10), respectively.

Expected downtime in a replacement cycle. Let $W$ be the downtime of the system in a replacement cycle. Then

$$
\begin{aligned}
\mathbb{E}\{W\}= & \mathbb{E}\left\{\left(T-\min \left(Y, S_{\mathrm{c}}\right) \mathbf{1}_{\left\{\min \left(Y, S_{\mathrm{c}}\right) \leq T\right\}}\right\}\right. \\
& +\sum_{k=2}^{\infty} \mathbb{E}\left\{\left(k T-\min \left(Y, S_{\mathrm{c}}\right)\right) \mathbf{1}_{\left\{(k-1) T<S_{\mathrm{p}},(k-1) T<\min \left(Y, S_{\mathrm{c}}\right) \leq k T\right\}}\right\} \\
= & \int_{0}^{T}\left(T-f_{\min \left(Y, S_{\mathrm{c}}\right)}(t)\right) \mathrm{d} t+\sum_{k=2}^{\infty}\left(\int_{(k-1) T}^{k T} \mathrm{~d} u \int_{(k-1) T}^{u} f_{2}(t, u)[k T-t] \mathrm{d} t\right. \\
& +\int_{(k-1) T}^{k T} \mathrm{~d} u \int_{u}^{k T} f_{1}(t, u)[k T-t] \mathrm{d} t \\
& \left.+\int_{k T}^{\infty} \mathrm{d} u \int_{(k-1) T}^{k T} f_{2}(t, u)[k T-t] \mathrm{d} t\right),
\end{aligned}
$$

where $f_{1}$ and $f_{2}$ are given by (8). 
Let $C\left(T, \xi_{\mathrm{p}}\right)$ be the expected cost rate for this maintenance model. By Tijms (2003),

$$
C\left(T, \xi_{\mathrm{p}}\right)=\frac{\mathbb{E}\{C\}}{\mathbb{E}\{R\}}
$$

where $\mathbb{E}\{C\}$ denotes the expected cost in a replacement cycle and $\mathbb{E}\{R\}$ denotes the expected time to a replacement cycle. So,

$$
C\left(T, \xi_{\mathrm{p}}\right)=\frac{C_{\mathrm{c}} \sum_{k=1}^{\infty} \mathbb{P}_{\mathrm{c}}\{k T\}+C_{\mathrm{p}} \sum_{k=1}^{\infty} \mathbb{P}_{\mathrm{p}}\{k T\}+C_{\mathrm{I}} \mathbb{E}\{N\}+C_{\mathrm{d}} \mathbb{E}\{W\}}{\mathbb{E}\{R\}},
$$

where $\mathbb{P}_{\mathrm{p}}\{k T\}, \mathbb{P}_{\mathrm{c}}\{k T\}, \mathbb{E}\{R\}, \mathbb{E}\{N\}$, and $\mathbb{E}[W]$ are given by (9)-(13), respectively.

The search of the optimal maintenance strategy is reduced to find the values $T^{\text {opt }}$ and $\xi_{\mathrm{p}}^{\text {opt }}$ such that

$$
C\left(T^{\mathrm{opt}}, \xi_{\mathrm{p}}^{\mathrm{opt}}\right)=\inf _{\left\{T>0,0<\xi_{\mathrm{p}} \leq \xi_{\mathrm{c}}\right\}} C\left(T, \xi_{\mathrm{p}}\right)
$$

where $C\left(T, \xi_{\mathrm{p}}\right)$ is given by (14). Due to the analytical complexity of $C\left(T, \xi_{\mathrm{p}}\right)$, the optimization of the expected cost rate for a data set is performed in the next section using numerical methods.

\section{Numerical example}

We consider a system subject to a random external shock process with intensity $\frac{1}{100}$ shocks per unit time. These shocks provoke a catastrophic failure with probability 0.05 or, otherwise, they increase the catastrophic failure rate by $\eta=\frac{1}{1000}$. We also assume that the baseline rate function of the catastrophic failure is given by $r_{0}(t)=\frac{1}{200}$ failures per unit time. Each shock, in its arrival, initiates a degradation process. The deterioration level of this degradation process is modelled by a gamma process with parameters $\alpha=1$ and $\beta=3$. Then $X_{v}(t)$, the deterioration level at $t$ time units after the initiation point $v$, follows a gamma distribution with parameters $t$ and 3, having the corresponding PDF

$$
f_{X_{v}(t)}(x)=\frac{3^{t}}{\Gamma(t)} x^{t-1} \mathrm{e}^{-3 x}, \quad x \geq 0
$$

for $t \geq 0$. The cost of a corrective replacement is $10 \mathrm{MU}$, the cost of a preventive replacement is $8 \mathrm{MU}$, the cost of an inspection is $0.005 \mathrm{MU}$ and the downtime cost is $0.5 \mathrm{MU}$ per unit time.

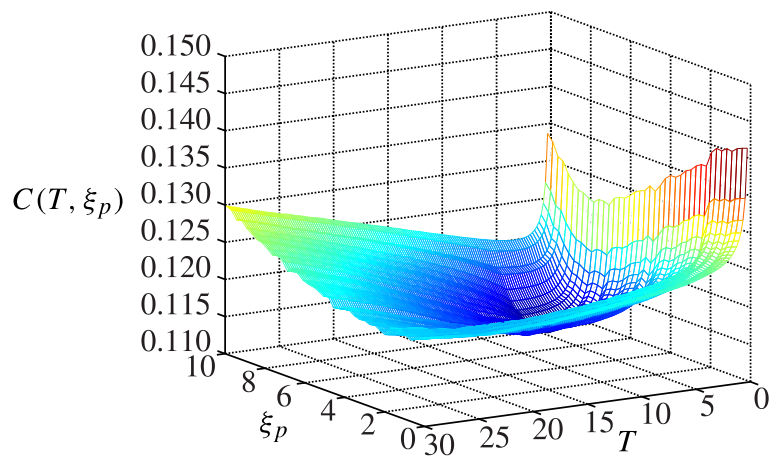

FIGURE 1: Expected cost rate versus $\xi_{\mathrm{p}}$ and $T$. 
In Figure 1, we show the expected cost rate versus $T$ and $\xi_{\mathrm{p}}$. The values of this figure have been calculated using a Monte Carlo simulation with 150 values of $T$ and 40 values for $\xi_{\mathrm{p}}$ and 60000 simulations for each pair of values. The optimal values for $T$ and $\xi_{\mathrm{p}}$ are given by $T^{\mathrm{opt}}=5.9$ and $\xi_{\mathrm{p}}^{\mathrm{opt}}=7.6923$ with an optimal expected cost rate of $C\left(T^{\mathrm{opt}}, \xi_{\mathrm{p}}^{\mathrm{opt}}\right)=0.110$ MUs per unit time.

\section{Concluding remarks}

In this paper we have studied a stochastic failure model for a system under a random shock environment with 'stochastically dependent' competing risks and developed a preventive maintenance policy for it. The conditional properties of the two competing risks have been discussed, and the basis for the development of the preventive maintenance policy has been built. Even though the preventive maintenance strategy considered in this paper utilizes only the information which is directly related with the Type II (degradation failure), it also contributes to the prevention of an unexpected failure by the Type I (catastrophic failure) due to the stochastic dependence of the two types. This is a new insight into the maintenance policy for a system with dependent competing risks which can generally be applied to the development of similar maintenance models under dependency. While the maintenance of the systems with 'independent competing risks' or with very simple dependency structure has been studied under specific settings in the literature, this paper is the first to consider the dependent competing risks model sharing external shocks under a general setting, and to provide new insights and stochastic interpretation on the dependency structure of the model from a theoretical point of view.

As mentioned above, this paper is based on a theoretically advanced stochastic model and relevant analysis in studying the lifetime of a system under a dynamic random environment. The theoretical basis for the maintenance policy and new insight into the structure of the stochastic dependency of the model is be one of the main contributions of this paper. Furthermore, since all the results have been obtained without specifying the degradation process, the results obtained in this paper may be regarded as very general and fundamental.

\section{Acknowledgements}

The corresponding author of this paper is Ji Hwan Cha. The authors thank the anonymous referee for very helpful detailed comments, which have improved the presentation and readability of this paper substantially. This work was supported by the National Research Foundation of Korea (NRF) grant funded by the Korea government (MEST) (grant no. 20110017338). This work was also supported by Priority Research Centers Program through the National Research Foundation of Korea (NRF) funded by the Ministry of Education, Science and Technology (grant no. 2009-0093827). For the second author, this research was supported by Ministerio de Economía y Competitividad, Spain (Project MTM2012-36603-C02-01), and Project GRU10110 from Gobierno de Extremadura, Spain.

\section{References}

Aven, T. (1996). Condition based replacement policies — a counting process approach. Reliab. Eng. System Safety 51, $275-281$.

Aven, T. And Jensen, U. (1999). Stochastic Models in Reliability. Springer, New York.

Bogdanoff, J. L. and Kozin, F. (1985). Probabilistic Models of Cumulative Damage. John Wiley, New York.

Castanier, B., Bérenguer, C. And Grall, A. (2003). A sequential condition-based repair/replacement policy with non-periodic inspections for a system subject to continuous wear. Appl. Stoch. Models Business Industry 19, 327-347. 
CHA, J. H. (2001). Burn-in procedures for a generalized model. J. Appl. Prob. 38, 542-553.

Ebrahimi, N. (1997). Multivariate age replacement. J. Appl. Prob. 34, 1032-1040.

Finkelstein, M. And Cha, J. H. (2013). Stochastic Modeling for Reliability. Shocks, Burn-in and Heterogeneous Populations. Springer, London.

Grall, A., Dieulle, L., Bérenguer, C. and Roussignol, M. (2002). Continuous-time predictive-maintenance scheduling for a deteriorating system. IEEE Trans. Reliab. 51, 141-150.

Kalbfleisch, J. D. and Prentice, R. L. (1980). The Statistical Analysis of Failure Time Data. John Wiley, New York.

KebIR, Y. (1991). On hazard rate process. Naval Res. Logistics 38, 865-876.

Lehmann, E. L. (1966). Some concepts of dependence. Ann. Math. Statist. 37, 1137-1153.

Liao, H., Elsayed, E. A. ANd Chan, L.-Y. (2006). Maintenance of continuously monitored degrading systems. Europ. J. Operat. Res. 175, 821-835.

Mi, J. (1994). Burn-in and maintenance policies. Adv. Appl. Prob. 26, 207-221.

NaKagawa, T. (2005). Maintenance Theory of Reliability. Springer, London.

PARK, C. AND PADGETt, W. J. (2006). Stochastic degradation models with several accelerating variables. IEEE Trans. Reliab. 55, 379-390.

Sherif, Y. S. AND Smith, M. L. (1981). Optimal maintenance models for systems subject to failure-a review. Naval Res. Logistics Quart. 28, 47-74.

Tisms, H. C. (2003). A First Course in Stochastic Models. John Wiley, Chichester.

VAldeZ-Flores, C. AND Feldman, R. M. (1989). A survey of preventive maintenance models for stochastically deteriorating single-unit systems. Naval Res. Logistics 36, 419-446.

WANG, H. (2002). A survey of maintenance policies of deteriorating systems. Europ. J. Operat. Res. 139, 469-489. 